\title{
Gravitational Kaluza-Klein modes in the String-Cigar Braneworld
}

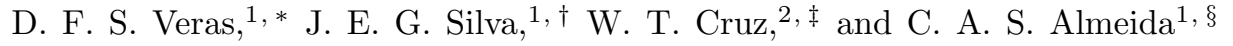 \\ ${ }^{1}$ Departamento de Física - Universidade Federal do Ceará (UFC) \\ C.P. 6030, 60455-760 Fortaleza-Ceará-Brazil \\ ${ }^{2}$ Instituto Federal de Educação, Ciência e Tecnologia do Ceará (IFCE) \\ Campus Juazeiro do Norte - CEP 63040-540, Juazeiro do Norte, Ceará , Brazil \\ (Dated: June 14, 2021)
}

\begin{abstract}
In this work the gravitational Kaluza-Klein (KK) modes are analysed on string-like braneworlds in six dimensions, namely the thin string-like Gherghetta-Shaposhnikov (GS) and the thick string-cigar model. We find a new massless mode in both modes satisfying the respective Schrödinger equation. By means of numerical analysis for both models, a complete graviton spectrum and eigenfunctions is attained. Besides the linear regime, the KK spectrum exhibits a decreasing behavior with an asymmetric mass gap with respect to the massless mode. The discontinuity is bigger in the GS model, and it is invariant upon the geometrical flow. A massive mode is obtained in the GS model with a tiny mass which does not match in any regime. It turns out that in the string-cigar model, the core brane structure smoothes and amplifies the KK modes near the brane. Further, the presence of a potential well allows the existence of resonant massive gravitons for small masses in the string-cigar scenario.
\end{abstract}

PACS numbers: 11.10.Kk, 11.27.+d, 04.50.-h, 12.60.-i

\footnotetext{
*Electronic address: franklin@fisica.ufc.br

${ }^{\dagger}$ Electronic address: euclides@fisica.ufc.br

‡Electronic address: wilami@pq.cnpq.br

§Electronic address: carlos@fisica.ufc.br
} 


\section{INTRODUCTION}

Among the high energy theories proposed in the last years, the braneworld models gained prominence due their fundamental basis on string-theory and since they grant an explanation for the gauge hierarchy and the cosmological constant problems [1 3. Once Kaluza-Klein (KK) models [4, 5] launched the possibility of extra dimensions, it has been blossomed models where the four-dimensional observable space-time is regarded as a membrane embedded in a higher-dimensional space-time [6]. The Randall-Sundrum (RS) model enhanced this hypothesis by assuming an infinite extra dimension whose warped geometry yields to a compact transverse space [2]. In the sequel, some models appeared proposing the brane be generated by a topological defect. In five dimensions, domain walls have been used to represent the brane [7, 9, 10, whilst in six dimensions, with axial symmetry the geometry resemblances that of the cosmic strings [1]. The resulting scenario is the so-called string-like braneworld [12].

Systems composed by complex scalar field (global) and gauge field (local) often describe string-like objects, such as the vortices and the cosmic strings [13. However, unlike the domain walls, there is no complete (interior and exterior) string-like solution analytically known, even in flat space-time. In warped braneworlds, Cohen-Kaplan [14, Gregory [15] and Olaganesi-Vilenkin [16] studied the exterior solution of a global string-like brane with and without bulk cosmological constant. The local case was addressed numerically by Giovaninni et al which acquired a smooth geometry satisfying the dominant energy condition [17.

Apart from the particular model used to compose the brane core, the exterior warped string-like solution with cosmological constant has a conformally-flat behavior that extends the five-dimensional Randall-Sundrum model [18]. In the infinitely thin core brane limit, the resulting model is called the Gherghetta-Shaposhnikov (GS) model [19]. The GS model provides a correction to the Newtonian potential due to the KK modes less than in the RS model [19. Another advantage over the RS model is that the Kaluza-Klein massless mode of the gauge field is naturally localized on the string-brane without any further coupling [20, 21]. The fermionic field, in its turn, can be trapped in the GS brane through a gauge minimal coupling only 20,22 .

Besides the interesting features for the fields, the string-like braneworlds also exhibit a rich geometric structure. In fact, the two extra dimensions form a transverse manifold with its internal symmetries and properties that reflect on the brane tension and geometry [12. The transverse manifold used in the GS model is a disc whereas Kehagias proposed a conical space to provide an explanation for the cosmological constant problem [23. Garriga-Porrati studied the effects generated by a football-shaped manifold [24] and Gogberashvili et al addressed the fermion generations problem by means of an apple-shaped space 25. In a supersymmetry setup, de Carlos and Moreno found a gravity trapping solution without cosmological constant and that asymptotically has a constant transverse radius, named as a cigar-like universe [26].

In a recent work published by two of the present authors [27, the so-called cigar soliton is used to construct an interesting smooth interior and exterior string-like geometry referred as the string-cigar. The cigar soliton is a twodimensional self-similar solution of the Ricci flow, a geometric flux driven by the Ricci tensor [28, 29. There are important applications of the Ricci flow in differents branches of Physics as in the sigma models 30, Euclidean black holes [31, topological massive gravity [33], ADM mass [32] and in the Heisenberg model in statistical mechanics [34]. The Ricci flow defines a family of geometries developing under an evolution parameter $c$. Therefore, the string-cigar scenario proves the changes of the brane properties due to the geometric flow [36. Since the Ricci soliton extends the Einstein manifolds, the string-cigar geometry can be realised as an augmented codimension two model proposed by Randjbar-Daemi and Shaposhnikov whose transverse space is a Ricci flat manifold [35].

Another smoothed thick string-like model which leads to a geometrical flow in the transverse space was built with a section of the resolved conifold; an important orbifold in string theory 36. Both models yield to regular geometries that asymptotically recover the GS model [27]. Nevertheless, the string-cigar model satisfies all the regularity conditions required to ensure a well-behaved 3-brane at the origin whereas the resolution parameter plays the role of the radius of the fifth dimension what violates the conical behavior near the origin [36].

The string-cigar scenario enables the existence of a localized gravitational massless-mode which effectively describes the gravity on the brane [27. The massless (or zero) mode in this scenario has the same asymptotically exponential behavior of that in the GS model, but near the origin it is smooth and has a bell-shape. Hence, the string-cigar scenario smoothes the geometry and the zero-mode near the origin. As the geometry undergoes the Ricci flow in the transverse space, the massless mode changes its width and amplitude [27]. In this work, we find a new massless mode for the GS and the string-cigar models by the Schrödinger approach which exhibits a more localized behavior.

Another noteworthy feature of the string-cigar model is its inhomogeneous source [27. Indeed, the maximum of the stress-energy tensor components is displaced from the origin what suggests the brane core is shifted [27. Giovaninni et al found a similar behavior for an Abelian vortex with higher winding number 17. The gravitational massless mode shares the same profile, and it shows the influence of the geometric changes on the physics of the brane [27]. Moreover, as pointed out by Tinyakov and Zuleta, the source for the GS model does not satisfy the dominant energy condition 37. On the other hand, the string-cigar source undergoes a phase transition whereupon some configurations 
fulfill all the energy conditions [27. Further, as higher the bulk cosmological constant closer to the GS model is the string-cigar source.

Nonetheless, the complexity of the differential equation for the KK modes turns the numerical analysis the most subtle approach to obtain the whole spectrum of masses and eigenfunctions [40. Thus, the foremost purpose of this work is to attain the massive KK spectrum and to analyse how it behaves upon the Ricci flow that the cigar undergoes.

Although Gherghetta and Shaposhnikov have found a complete set of eigenfunctions for their thin-string model, the corresponding KK masses expression for the discrete states is valid only for large values of a discretization index $n$ [19. In this limit, the masses increase linearly but for small $n$ the behavior is yet unknown. Then, our numerical analysis provides the full KK mass spectrum of the GS and string-cigar scenarios. Both models show a decreasing behavior for small $n$ with a mass gap between the two patterns. Moreover, the GS model presents a massive mode with tiny mass and amplitude which is absent in the string-cigar model. The parallel between the two models provides more information of how the fields behave on singular and smooth scenarios in six dimensions. Giovaninni et al have numerically studied the interior and exterior geometry of a string-like braneworld, but have not concerned with the KK spectrum [17. In this article, numerical methods are also used to find and to study gravitational resonant massive modes on both GS and string-cigar models. Even though the analogous Schröedinger potential exhibits an infinite potential well around the origin for the string-cigar model, resonant modes are allowed in contrast with the GS model where they are absent. It turns out that the resonant modes appear only for small masses.

This paper is organized as follows: in the section II the main characteristics and results of the GS and string-cigar models are reviewed. Next, in section III, the numerical results which concern to the spectrum and eigenfunction are presented. In section IV, the study of KK massive modes as resonant states is developed. Finally, in the section $\mathrm{V}$, some final remarks and perspectives are outlined.

\section{STRING-LIKE BRANEWORLDS}

Consider a six dimensional space-time $\mathcal{M}_{6}$ built from the warped product between a $4 \mathrm{D}$ Lorentzian manifold $\mathcal{M}_{4}$ and the 2D Riemannian manifold $\mathcal{M}_{2}$. Hereinafter, we refer $\mathcal{M}_{4}$ as a 3 -brane and $\mathcal{M}_{2}$ as the transverse space.

A string-like braneworld is a static $6 \mathrm{D} \mathcal{M}_{6}$ endowed with an axial symmetry in the transverse space. A subtle metric for this model is given by [12, 14, 17, 19, 20,

$$
d s_{6}^{2}=g_{A B}(x, \rho, \theta) d x^{A} d x^{B}=\sigma(\rho) g_{\mu \nu}(x) d x^{\mu} d x^{\nu}-d \rho^{2}-\gamma(\rho) d \theta^{2},
$$

where $x$ are brane coordinates, $\rho \in[0, \infty)$ and $\theta \in[0,2 \pi]$ are the extra dimensions and $\sigma$ and $\gamma$ are the so-called warp factors.

The additional regularity conditions,

$$
\begin{aligned}
& \sigma(0)=1, \sigma^{\prime}(0)=1, \\
& \gamma(0)=0, \quad(\sqrt{\gamma(0)})^{\prime}=1,
\end{aligned}
$$

are imposed in order to avoid singularities [12, where the $\left({ }^{\prime}\right)$ stands for the derivative $\partial_{\rho}$. The conditions for $\sigma$ in Eq. (2) are already present in the RS models [1, 2] whereas the further assumption for $\gamma$ reflects the smooth but conical behavior near the origin [12, 17, 19, 37.

Regardless the particular model for the source, an axisymmetric stress-energy tensor of the following form is adopted 12, 17, 19,

$$
T_{B}^{A}=\operatorname{diag}\left(t_{0}, t_{0}, t_{0}, t_{0}, t_{\rho}, t_{\theta}\right) .
$$

For a global string, for instance, $t_{\rho}=-t_{\theta}$ [14-16]. In the presence of a bulk cosmological constant $\Lambda$ and for a flat brane $\mathcal{M}_{4}$, the Einstein equation reads

$$
R_{a b}-\frac{R}{2} g_{a b}=-\kappa_{6}\left(\Lambda g_{a b}+T_{a b}\right)
$$

that for the metric ansatz (1), yields to

$$
\begin{aligned}
\frac{3}{2}\left(\frac{\sigma^{\prime}}{\sigma}\right)^{\prime}+\frac{3}{2}\left(\frac{\sigma^{\prime}}{\sigma}\right)^{2}+\frac{3}{4} \frac{\sigma^{\prime}}{\sigma} \frac{\gamma^{\prime}}{\gamma}+\frac{1}{4}\left(\frac{\gamma^{\prime}}{\gamma}\right)^{2}+\frac{1}{2}\left(\frac{\gamma^{\prime}}{\gamma}\right)^{\prime} & =-\kappa_{6}\left(\Lambda+t_{0}(\rho)\right), \\
\frac{3}{2}\left(\frac{\sigma^{\prime}}{\sigma}\right)^{2}+\frac{\sigma^{\prime}}{\sigma} \frac{\gamma^{\prime}}{\gamma} & =-\kappa_{6}\left(\Lambda+t_{\rho}(\rho)\right), \\
2\left(\frac{\sigma^{\prime}}{\sigma}\right)^{\prime}+\frac{5}{2}\left(\frac{\sigma^{\prime}}{\sigma}\right)^{2} & =-\kappa_{6}\left(\Lambda+t_{\theta}(\rho)\right),
\end{aligned}
$$


where $\kappa_{6}$ is the six-dimensional gravitational constant related to the six-dimensional energy scale by $\kappa_{6}=\frac{8 \pi}{M_{6}^{4}}[27$.

Analysing the vacuum configuration, the equation

$$
2\left(\frac{\sigma^{\prime}}{\sigma}\right)^{\prime}+\frac{5}{2}\left(\frac{\sigma^{\prime}}{\sigma}\right)^{2}=-\kappa_{6} \Lambda
$$

determines the warp function. Defining

$$
y(\rho)=\frac{\sigma^{\prime}}{\sigma}
$$

the Eq. (8) turns to be

$$
y^{\prime}+\frac{5}{4} y^{2}-\frac{\kappa_{6}|\Lambda|}{2}=0
$$

whose solution is

$$
y(\rho)=c \tanh \frac{5 c}{4}\left(\rho+\rho_{0}\right)
$$

where

$$
c^{2}=-\frac{2 \kappa_{6}}{5} \Lambda
$$

and $\rho_{0}$ is an integration constant. Integrating Eq. 11] yields to the warp factor

$$
\sigma(\rho)=\sigma_{0} \cosh ^{\frac{4}{5}}\left(\frac{5}{4} c \rho\right)
$$

with a integration constant $\sigma_{0}$. The warp factor in 13 provides an infinite volume to the transverse space which leads to a non four-dimensional effective gravitational theory.

The relation between the bulk Planck mass $M_{6}$ and brane Planck masses $M_{4}$ is given by

$$
M_{4}^{2}=2 \pi M_{6}^{4} \int_{0}^{\infty} \sigma(\rho) \sqrt{\gamma(\rho)} d \rho .
$$

Then, for a string-like model with warp function 13 the brane Planck scale diverges.

Another important characteristic of the string-like models are the string tensions $\mu_{i}$ defined by [17, 19]

$$
\mu_{i}(c)=\int_{0}^{\epsilon} t_{i}(\rho, c) \sigma^{2}(\rho, c) \sqrt{\gamma(\rho, c)} d \rho,
$$

which determines the matching between an internal and an external geometric solution made in the boundary of the core with width $\epsilon[12,17,19]$.

Once presented the general aspects of the string-like scenario, the behavior of the small metric fluctuations around this configuration is given below.

Performing the following conformal invariant perturbation [17, 19, 27, 39]

$$
d s_{6}^{2}=\left(\sigma(\rho) \eta_{\mu \nu}+h_{\mu \nu}(x, \rho)\right) d x^{\mu} d x^{\nu}+d \rho^{2}+\gamma(\rho) d \theta^{2},
$$

the first-order perturbed Einstein equation (4) yields to [17, 19, 27, 39.

$$
\square_{6} h_{\mu \nu}=\partial_{A}\left(\sqrt{-g_{6}} \eta^{A B} \partial_{B} h_{\mu \nu}\right)=0 .
$$

Thus, the tensorial perturbation $h_{\mu \nu}$ can be regarded as a tensorial field (graviton) propagating in the bulk.

Assuming the usual Kaluza-Klein decomposition [2, 19, 20, 27.

$$
h_{\mu \nu}(x, \rho, \theta)=\sum_{l, m=0}^{\infty} \phi_{m, l}(\rho) \mathrm{e}^{i l \theta} \hat{h}_{\mu \nu}(x),
$$


and a free-wave dependence on the 3-brane

$$
\square_{4} h_{\mu \nu}\left(x^{\xi}\right)=m_{0}^{2} h_{\mu \nu}\left(x^{\xi}\right),
$$

the graviton equation of motion (17), for a conformal scenario where $\gamma(\rho)=\sigma(\rho) \beta(\rho)$, reads [19, 27]

$$
\left(\sigma^{\frac{5}{2}} \sqrt{\beta} \phi_{m, l}^{\prime}(\rho)\right)^{\prime}+\sigma^{\frac{3}{2}} \sqrt{\beta}\left(m_{0}^{2}-\frac{l^{2}}{\beta^{2}}\right) \phi_{m, l}(\rho)=0 .
$$

The function $\beta$ is responsible by the conical behavior [27, 36. The Eq. 20 describes the radial behavior of the graviton on string-like scenarios. The presence of the angular number $l$ turns the spectrum degenerated [19]. In addition, due to the axial symmetry, the boundary conditions are [17, 19, 27]

$$
\phi^{\prime}(0)=\phi^{\prime}(\infty)=0
$$

The radial equation (20) and the boundary conditions 21 provide a set of solutions whose orthogonality relation is given by

$$
\int_{0}^{\infty} d \rho \sigma^{\frac{3}{4}} \sqrt{\beta} \phi_{m, l} \phi_{n, l^{\prime}}=\delta_{m n} \delta_{l l^{\prime}}
$$

The eigenvalues of Eq. 20 satisfying the boundary conditions 21 are called the KK spectrum (mass) and the respective eigenfunctions are called the KK states. Among the KK states there is one for a vanishing mass, called massless or zero mode. From Eq. (20), the massless mode has the form

$$
\phi_{0}(\rho)=A_{1} \int_{0}^{\rho} \sigma^{-\frac{5}{2}} \beta^{-\frac{1}{2}} d \rho^{\prime}+A_{2},
$$

where $A_{1}$ and $A_{2}$ are constants. A similar massless mode was found by Csaki et al for non-conformally flat space-times 39 .

A suitable way to study the KK spectrum consists of turn Eq. 20 into a Schrödinger-like equation. By taking the change of independent variable

$$
z(\rho)=\int^{\rho} \sigma^{-1 / 2} d \rho^{\prime}
$$

and of a dependent variable

$$
\phi_{m}(z)=u(z) \Psi_{m}(z)
$$

where

$$
\frac{\dot{u}}{u}+\frac{\dot{\sigma}}{\sigma}+\frac{1}{4} \frac{\dot{\beta}}{\beta}=0
$$

the radial equation 20 yields to

$$
-\ddot{\Psi}_{m}(z)+U(z) \Psi_{m}(z)=m^{2} \Psi_{m}(z)
$$

with

$$
U(z)=\frac{\ddot{\sigma}}{\sigma}+\frac{1}{2} \frac{\dot{\sigma}}{\sigma} \frac{\dot{\beta}}{\beta}-\frac{3}{16}\left(\frac{\dot{\beta}}{\beta}\right)^{2}+\frac{1}{4} \frac{\ddot{\beta}}{\beta}+\frac{l^{2}}{\beta} .
$$

The boundary conditions 21 implies the following boundary condition for $\Psi(z)$ :

$$
\begin{aligned}
u^{\prime}(0) \Psi(0)+u(0) \Psi^{\prime}(0) & =0, \\
u^{\prime}(\infty) \Psi(\infty)+u(\infty) \Psi^{\prime}(\infty) & =0 .
\end{aligned}
$$

Besides the bijective relation between the Sturm-Liouville and the Schrödinger approaches, the last provides information about resonant modes that we consider in section (IV). In the follows, the geometrical features and the properties of Eq. 20 in the GS model and the string-cigar model are studied. 


\section{A. The GS model}

Gherghetta and Shaposhnikov found a vacuum solution of Einstein equations given in Eq. (4) that localizes the gravity on the string-like brane. Assuming that [19]

$$
\frac{\sigma^{\prime}}{\sigma}=-c
$$

which can be obtained from the hyperbolic tangent function in Eq. (11) in one of its asymptotic values, the Eq. 8) yields to the following warp function

$$
\sigma(\rho)=\mathrm{e}^{-c \rho}
$$

Moreover, for

$$
\beta(\rho)=R_{0}^{2},
$$

the GS model describes a $A d S_{6}$ space-time [19. Since the GS model is built from the vacuum solution, it can be regarded as the space-time of a thin string-like braneworld, i.e., $\epsilon \rightarrow 0$ [17, 37. In addition, the GS solution does not satisfy the regularity conditions at the origin (2).

In the GS model, the graviton obeys the radial equation [19]

$$
\phi_{m}^{\prime \prime}-\frac{5}{2} c \phi_{m}^{\prime}+\left(m_{0}^{2}-l^{2} / R_{0}^{2}\right) \mathrm{e}^{c \rho} \phi_{m}=0,
$$

which general solution, can be written in terms of the Bessel functions

$$
\phi_{m}(\rho)=\mathrm{e}^{\frac{5}{4} c \rho}\left[B_{1} J_{5 / 2}\left(\frac{2 m}{c} \mathrm{e}^{\frac{1}{2} c \rho}\right)+B_{2} Y_{5 / 2}\left(\frac{2 m}{c} \mathrm{e}^{\frac{1}{2} c \rho}\right)\right]
$$

where $B_{1}$ and $B_{2}$ are arbitrary constants and $m=m_{0}^{2}-l^{2} / R_{0}^{2}$. This solution grows exponentially revealing that massive modes are not localized on the brane [19].

From Eq. 23], the GS massless mode has the form

$$
\phi_{0}(\rho)=A_{1} \mathrm{e}^{\frac{5}{2} c \rho}+A_{2}
$$

Among the two solutions in Eq. (35), only the $\phi_{0}=A_{2}$ satisfies the orthogonality relation (22) [19]. Gherghetta and Shaposhnikov defined an orthonormal solution by [19]

$$
\psi_{m}(\rho)=\mathrm{e}^{-\frac{3}{4} c \rho} \phi_{m}(\rho)
$$

so that, the zero-mode becomes

$$
\psi_{0}(\rho)=\sqrt{\frac{3 c}{2 R_{0}}} \mathrm{e}^{-\frac{3}{4} c \rho} .
$$

Therefore, a massless mode is localized in the thin-string brane [19. However, this solution does not satisfy the boundary conditions (21) at $\rho=0$, because the warp factor (31) does not obey the usual regularity conditions. On the other hand, by means of the Schrödinger approach, another more localized massless mode was found. Indeed, the Schrödinger equation (27) for the GS model is given by

$$
-\ddot{\Psi}_{m}+\frac{6}{z^{2}} \Psi_{m}=m^{2} \Psi_{m}
$$

where $z=\frac{2}{c} \mathrm{e}^{\frac{c}{2} \rho}$ and by Eq. 26, , the relation between $\phi_{m}$ and $\Psi_{m}$ is

$$
\phi_{m}=C_{0} \mathrm{e}^{c \rho} \Psi_{m}
$$

For $m=0$, Eq. 38 has the solutions

$$
\begin{aligned}
\Psi_{0} & =C_{1} z^{3}+C_{2} z^{-2} \\
& =C_{1} \mathrm{e}^{\frac{3}{2} c \rho}+C_{2} \mathrm{e}^{-c \rho}
\end{aligned}
$$


which using Eq. (39) yields to the zero-mode (35). In order to $\Psi_{0}$ be normalized, we set $C_{1}=0$. Therefore, a localized zero-mode satisfying the analogous Schrödinger equation in the GS model is given by

$$
\Psi_{0}(\rho)=\sqrt{\frac{7 c}{2 R_{0}}} \mathrm{e}^{-c \rho} .
$$

Thus, the zero-mode $\Psi_{0}$ in Eq. 41 has a bigger value at the origin and a bigger decay rate compared with $\psi_{0}$ proposed in Ref. [19. Further, the massless mode (41) satisfies the boundary conditions (29).

For $m \neq 0$, the solution of GS schrodinger equation is

$$
\Psi_{m}(z)=\sqrt{\frac{2}{m \pi}}\left(\frac{1}{m z}\right)^{2}\left[\left(m^{2} z^{2}-3 m z-3\right)(\cos (m z)-\sin (m z))\right] .
$$

Since $\Psi_{m}(z)$ is not defined for $m=0$, we can not obtain the massless mode 41 from Eq. 42. This shows a mass gap in GS model which we explore numerically in section (III). Moreover, in the asymptotic limit the massive modes $\Psi_{m}$ assume the plane wave form given by

$$
\Psi_{m}(z \rightarrow \infty)=\sqrt{\frac{2}{m \pi}}(\cos (m x)-\sin (m x)) .
$$

The KK spectrum can be obtained by inserting a finite radial distance cutoff $\rho_{\max }$ and imposing the boundary conditions (21). Then, the spectrum is obtained by the zeroes of the Bessel function

$$
J_{\frac{3}{2}}\left(\frac{2 m_{n}}{2} \mathrm{e}^{\frac{c}{2} \rho_{\max }}\right)=0
$$

that for large index $n$ yields to [19]

$$
m_{n} \simeq c\left(n-\frac{1}{2}\right) \frac{\pi}{2} \mathrm{e}^{-\frac{c}{2} \rho_{\max }} .
$$

The linear relation between the mass and the index $n$ is rather important in the Newtonian potential correction [19]. Nevertheless, the formula (45) is valid only for great $n$. In section (III), the eigenfunctions of the GS model and a complete spectrum $m_{n}$ is numerically obtained.

\section{B. The string-cigar model}

An extension to GS model, the so-called string-cigar braneworld [27, is built from a warped product between 3 -brane and the cigar soliton space. The cigar soliton is a two dimensional stationary solution for the Ricci flow

$$
\frac{\partial}{\partial \lambda} g_{a b}(\lambda)=-2 R_{a b}(\lambda)
$$

where $\lambda$ is a metric parameter and $R_{a b}$ is the Ricci tensor [28]. An axisymmetric metric for the cigar soliton reads as [29]

$$
d s_{\lambda}^{2}=d \rho^{2}+\frac{1}{\lambda^{2}} \tanh ^{2}(\lambda \rho) d \theta^{2} .
$$

It is straightforward to see that the cigar soliton is a smooth manifold.

The main idea of the string-cigar scenario is to use the cigar soliton as the transverse space in order to smooth the GS model. The Ricci flow defines a family of string-like branes and the evolution of the transverse space is related to variations on the geometric and physical quantities [27]. Since the asymptotic value of the scalar curvature depends on the evolution parameter [27, the geometric flow represents a variation of the bulk cosmological constant. Then, $\lambda$ and $c$ are equivalent labels. The string-cigar scenario is asymptotically flat as the disc of radius $1 / \lambda=R_{0}$ used in the GS model [28. However, near the origin, the $\tanh ^{2} \rho$ term smoothes the geometry and provides a thickness to the brane [27.

The warp factor and the angular metric component proposed are, respectively [27,

$$
\sigma(\rho, c)=\mathrm{e}^{-(c \rho-\tanh (c \rho))}
$$


and

$$
\gamma(\rho, c)=\frac{1}{c^{2}} \tanh ^{2}(c \rho) \sigma(\rho, c)
$$

The metric (1) with equations (48) and 49 represents a space-time inside and outside a string-like defect which satisfies all the regularity conditions given in Eq. (2) 27.

The Einstein equation provides the energy density [27]:

$$
t_{0}(\rho, c)=\frac{c^{2}}{\kappa_{6}}\left(7 \operatorname{sech}^{2}(c \rho)+\frac{13}{2} \operatorname{sech}^{2}(c \rho) \tanh (c \rho)-\frac{5}{2} \operatorname{sech}^{4}(c \rho)\right) .
$$

The Fig. 1 shows the energy density for some values of the evolution parameter $c$. The source is shifted from the origin, and the width $\epsilon$ can be estimated as $\epsilon=\bar{\rho}$, where $\bar{\rho}$ stands to the half-maximum position of $t_{0}$. Further, as more the value of $c$ less the width of the core $\epsilon$ and then, closer to the GS model is the string-cigar solution.

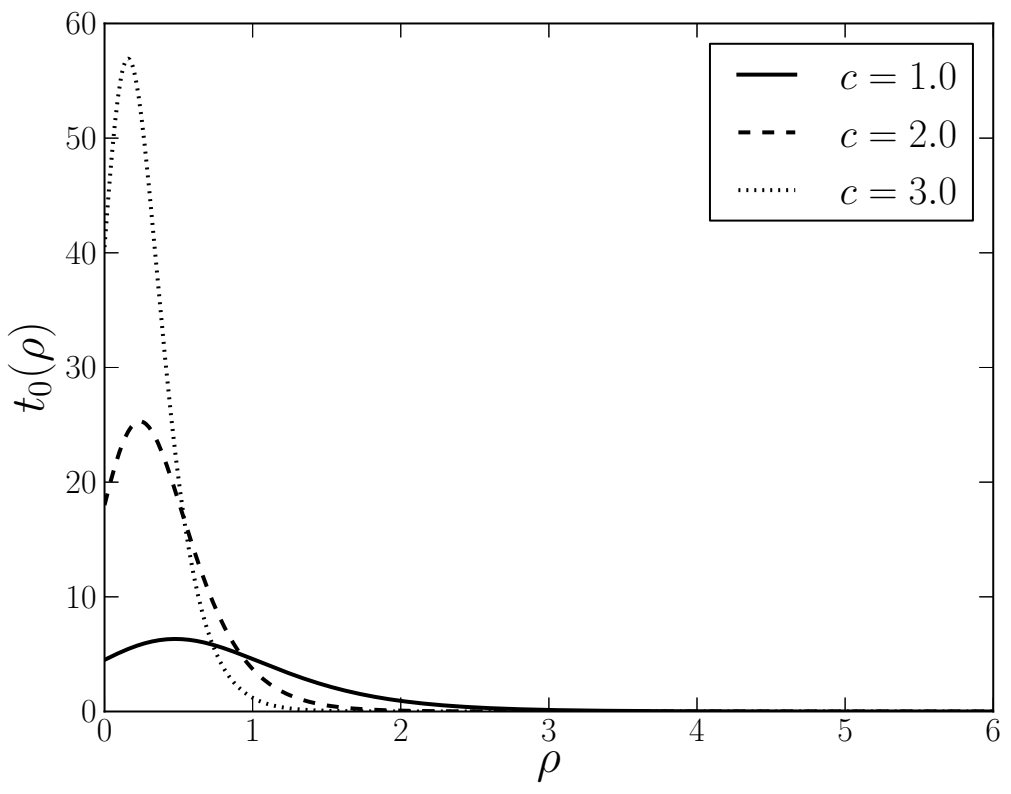

Figure 1: Energy density of the string-cigar braneworld.

In the string-cigar geometry, the radial KK equation reads

$$
\phi_{m}^{\prime \prime}+c\left[-\frac{5}{2} \tanh ^{2}(c \rho)+\frac{\operatorname{sech}^{2}(c \rho)}{\tanh (c \rho)}\right] \phi_{m}^{\prime}+\mathrm{e}^{(c \rho-\tanh (c \rho))}\left(m_{0}^{2}-\frac{l^{2} c^{2}}{\tanh ^{2}(c \rho)}\right) \phi_{m}=0 .
$$

Note that the mass term has a radial dependence which blows up at the origin and converges to the GS value asymptotically. For $\rho \rightarrow \infty$, the equation (51) assumes the same form as (33) with a re-scaled mass $m \rightarrow \mathrm{e}^{-1 / 2} m$. Hence, the asymptotic solutions of (51) have the same behavior of Eq. (34) 27].

A normalized zero-mode solution satisfying the Schrödinger equation for the string-cigar model, is defined as [27].

$$
\Psi_{0}(\rho, c)=N \sigma(\rho, c)\left(\frac{\tanh c \rho}{c}\right)^{1 / 4}
$$

where $N$ is a normalization factor. The solution (52) can be realized as a smoothed GS massless mode (35). In contrast with the GS model, the string-cigar massless mode vanishes at the origin because of the conical behavior. This agrees with the shift of the brane core.

However, the complexity of the radial equation (51) turns necessary the use of numerical methods to derive solutions for the complete domain. They are presented on section III] 


\begin{tabular}{|c|c|}
\hline$m_{454}=4.295$ & $m_{458}=0.317$ \\
\hline$m_{455}=5.172 \times 10^{-7}$ & $m_{459}=0.411$ \\
\hline$m_{456}=0.130$ & $m_{460}=0.504$ \\
\hline$m_{457}=0.224$ & $m_{461}=0.598$ \\
\hline
\end{tabular}

Table I: Some mass eigenvalues for high values of the discretization index where the linear regime (for $n>455)$ is noticiable. The smallest mass value $m_{455}$ corresponds to the transient state.

\section{MASS SPECTRUM AND KK EIGENFUNCTIONS}

We solved the equations (33) and (51) by the matrix method [45] with second order truncation error in order to attain the complete KK spectrum. Since the angular number $l$ leads to a degenerate spectrum, $l=0$ solutions (which is referred to s-waves) were searched. Henceforward, we will label the graviton mass as $m$, instead of $m_{0}$. Further, these Sturm-Liouville problems are extremely sensible on $c$ parameter variations due to the exponential term. Then, to prevent overflow errors, we fixed $c=1.0$. For the GS model, the domain $\rho$ was discretized in $[0.0,5.7]$ with $N=500$ uniform subdivisions. However, since Eq. (51) is singular at the origin, and the 1st (0th)-order derivative coefficient is strongly active for small (large) $\rho$ values, the optimum domain for the string-cigar model is $[0.01,5.70]$.

The number of subdivisions of the interval $\left[a, \rho_{\max }\right], n$, is intrinsically related with the number of the zeroes of the Bessel function $\tilde{n}$ in this range. In fact, as more $n$ more zeroes exist in the interval. These zeroes split the interval in a partition. Therefore, the eigenvalues $m_{n}$ are labelled by the number of subdivisions. Henceforward, $n$ and $\tilde{n}$ are equivalent labels.

The figures 2 and 3 show the complete spectrum $m_{n}$ for GS and string-cigar models, respectively. The eigenvalues are all real and thus, the models do not carry tachyons. For large values of the discretization index $n$ (on the present case, $n>455)$ the mass spectrum for both models grows linearly, what agrees with the GS model 19 . On the other hand, for $n \leq 400$, the mass values decrease as $m_{n} \approx \frac{1}{n}$, an important new behavior that can leads to corrections on the Newtonian potential [19. The decreasing behavior for small $n$ matches with the analytical expression for the zeros of the Bessel functions $J_{\frac{3}{2}}\left(\frac{2 m_{n}}{c} \mathrm{e}^{\frac{1}{2} c \rho}\right)=0$, namely [38]

$$
m_{n}=\frac{c}{2}\left[\left(n-\frac{1}{2}\right) \pi+\frac{1}{4\left[\left(n-\frac{1}{2}\right) \pi\right]}+\ldots\right] \mathrm{e}^{-\frac{c \rho}{2}} .
$$

Hence, for small $n$, the decreasing behavior prevails over the linear regime.

For $n=455$, the minimum mass value is $m_{455}=5.172 \times 10^{-7}$, rather small compared to the whole set, whose respective eigenfunction $\phi_{455}$ is plotted in Fig. 4. This eigenfunction possesses a tiny amplitude and shares a similar profile of the GS localized massless mode. Such feature can be reported as a transient state. However, unlike the GS zero mode, $\phi_{455}$ satisfies the boundary conditions $(21)$.

The numerical analysis revealed another feature of the GS model not present in the Ref. 19], a mass gap between the decreasing and the linear regimes. It turns out that there are two asymmetric mass gaps on GS model between the transient state $m_{455}$ and its neighbours. Graphically, the gap between the decreasing regime and the transient state is clearly noticeable. The table (I) shows that the separation between $m_{455}$ and $m_{456}$ is higher than the subsequent ones that are approximately $\Delta m \approx 0.094$. Therefore, the transition between the decreasing and linear regimes is not continuous and passes through a transient state of tiny mass.

The existence of a mass gap and the transient massive mode agrees with the analytical solutions for the massive modes (34) and for the massless mode (35). Indeed, for small masses it can expands the massive mode as

$$
\phi_{m \rightarrow 0}(\rho)=D_{1}\left(\frac{c}{2 m}\right)^{\frac{5}{2}}+D_{2}\left(\frac{c}{2 m}\right)^{\frac{1}{2}} \mathrm{e}^{c \rho}+D_{3}\left(\frac{2 m}{c}\right)^{\frac{1}{2}} \mathrm{e}^{\frac{3}{2} c \rho}+\mathcal{O}\left(\mathrm{e}^{\frac{5}{2} c \rho}\right),
$$

where $D_{1}, D_{2}$ and $D_{3}$ are constants determined by the constants $B_{1}$ and $B_{2}$ and by the expansion of the Bessel functions at the origin. It is worthwhile to say that since $\lim _{m \rightarrow 0} \phi_{m}(\rho)=+\infty$, it is not possible to attain continuously the massless mode (35) from the massive mode (34). Thus, there is a mass gap between the massless and the first massive modes. Further, the small amplitude of $\phi_{455}$ can be explained by the small values of the constants, e.g., $D_{3} \approx 10^{-32}$, and by the boundary conditions which relate the constants. 
The string-cigar mass spectrum is very similar to the GS one except by the absence of the tiny transient massive mode as in GS model. The minimum mass value is far from zero. Thus, the near brane resolution driven by the string-cigar geometry avoids this transient mode, and it becomes more regular the massive spectrum. Furthermore, it turns out that the mass gap remains invariant for all the values of $c$ used. Then, this suggests that the present geometrical flow do not break the discontinuity between the two regimes.

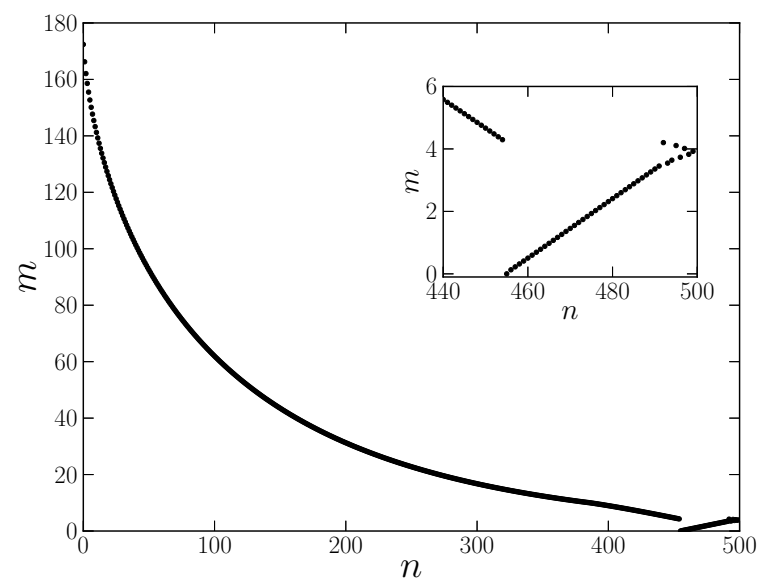

Figure 2: Thin string model's mass spectrum obtained by the matrix method. The sub-graph is the linear regime's scale magnification. The last points are numerical noises.

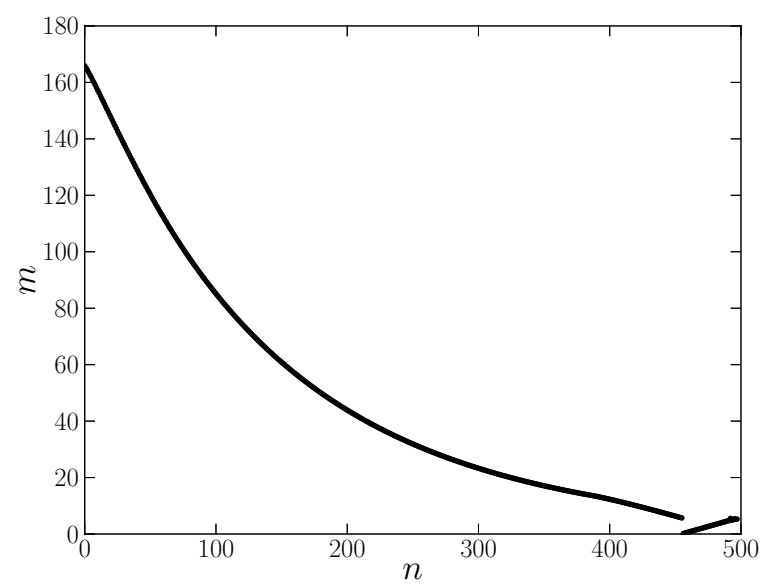

Figure 3: Mass spectrum of the string-cigar model obtained by matrix method. It is very similar to the GS model except by the absence of a transient state.

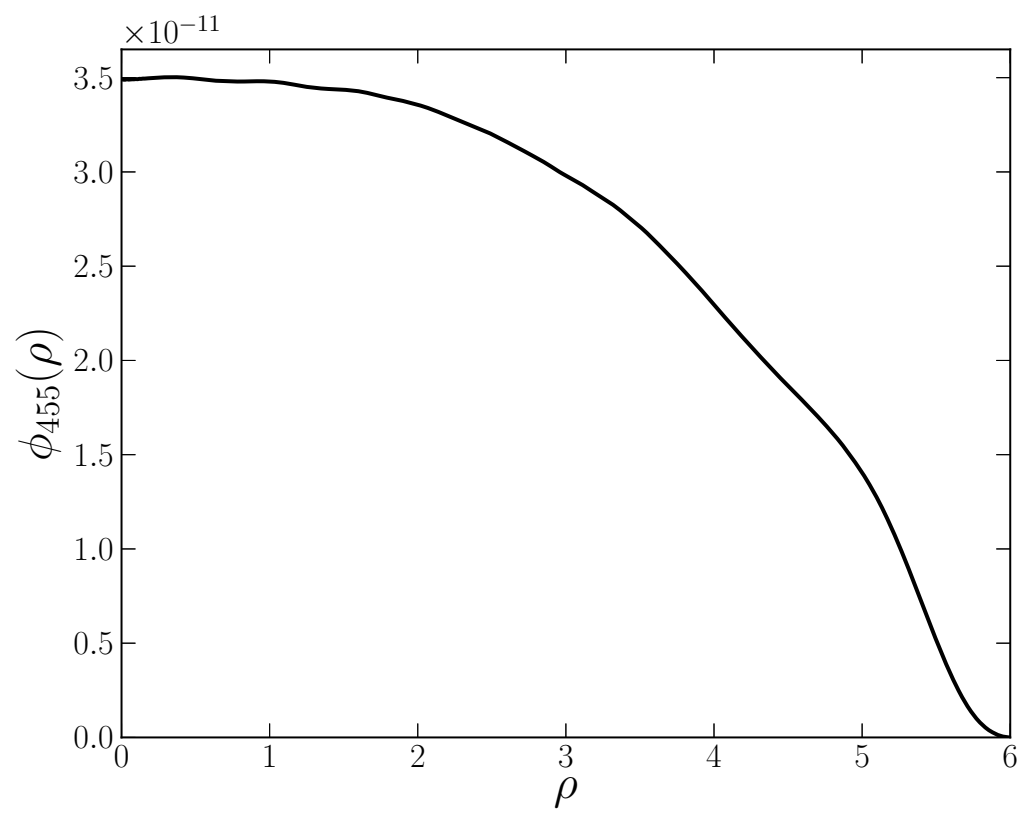

Figure 4: GS model's numerical eigenfunction for $m=5.172 \times 10^{-7}$.

The GS and the string-cigar eigenfunctions were plotted for some mass eigenvalues in the Figs. $5,6,6$ and 8 , respectively. As expected, the massive solutions for both models match the same behavior asymptotically. Near the origin, the massive modes of the string-cigar model have a bigger amplitude compared with those of the GS model. Moreover, they are smooth in the core and near the brane as predicted in Ref. [27]. At this limit, the string-cigar eigenfunctions behave as Bessel functions of first kind $J_{0}(m \rho)$. Indeed, a first-order expansion of the coefficients of Eq. (51) at the origin yields to 


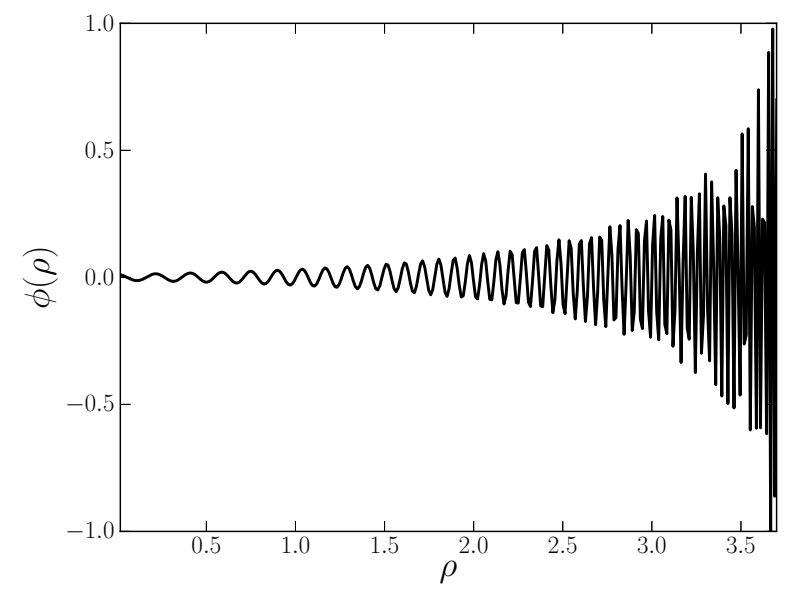

Figure 5: Thin string GS model's numerical eigenfunction for $m=37.156$.

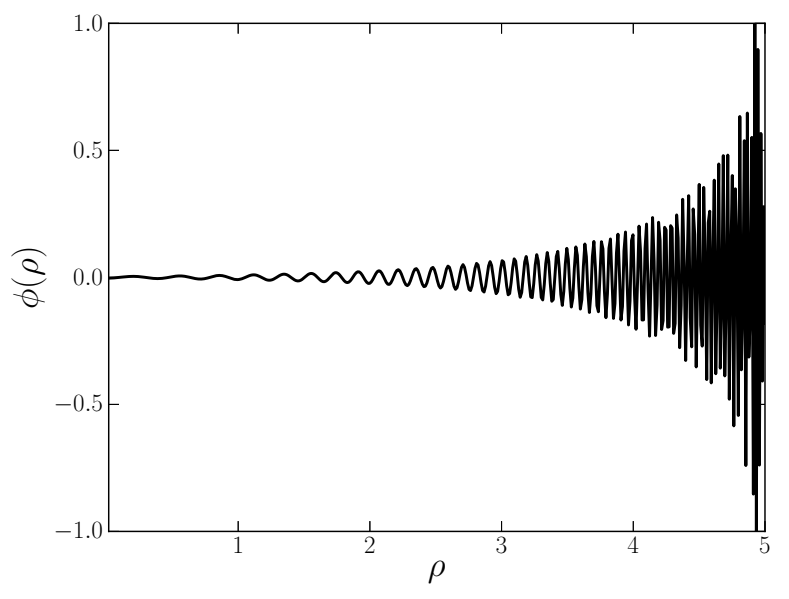

Figure 6: Thin string GS model's numerical eigenfunction for $m=22.729$.

$$
\phi_{m}^{\prime \prime}+\left(\frac{1}{\rho}-\frac{2}{3} c^{2} \rho\right) \phi_{m}^{\prime}+m^{2} \phi_{m}=0 .
$$

For $\rho \approx 0$ the term $\frac{1}{\rho}$ prevails over the term $-\frac{2}{3} c^{2} \rho$ what yields to a Bessel equation whose solution is

$$
\phi_{\rho \rightarrow 0}(\rho)=E_{1} J_{0}(m \rho)+E_{2} Y_{0}(m \rho)
$$

where $E_{1}$ and $E_{2}$ are constants. Since $Y_{0}$ diverges at the origin, $E_{2}=0$, fitting the behavior sketched in Figs. 7 and 8 .

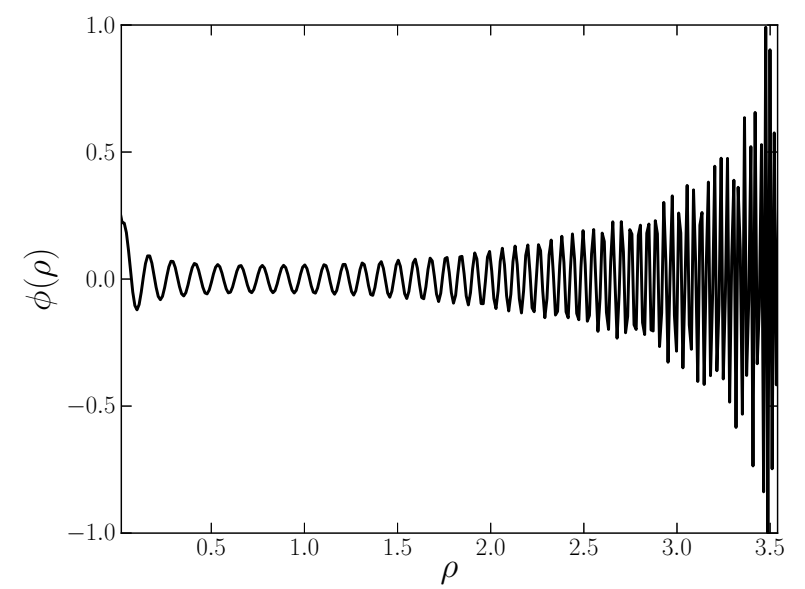

Figure 7: String-cigar model's numerical eigenfunction for $m=37.022$.

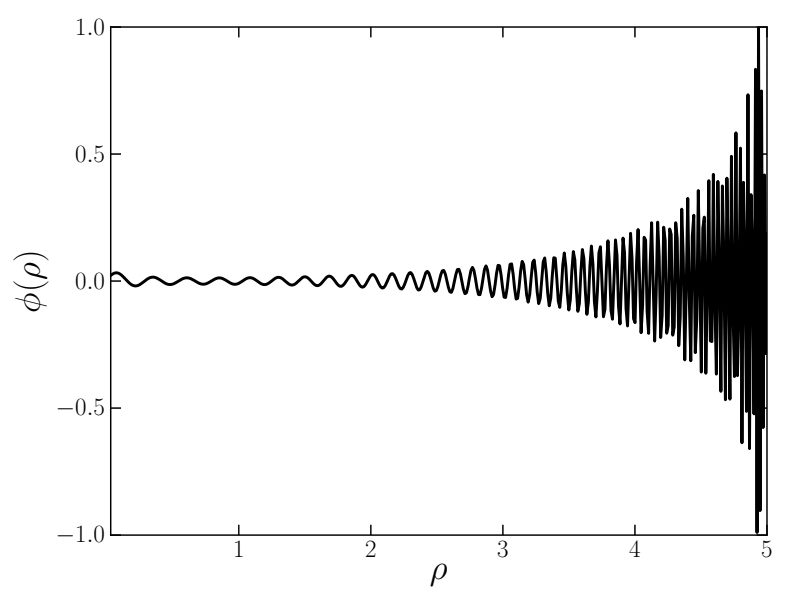

Figure 8: String-cigar model's numerical eigenfunction for $m=22.721$.

\section{KK RESONANT MODES}

Despite the divergent behavior of the massive gravitational modes, some of these modes can show a resonant profile [10, 42]. These states may be found by means of the resonance method [4] that provides solutions of the Schrödinger 
equation which exhibit large amplitudes near the brane in comparison with its values far from the defect. Large peaks in the distribution of the wave function in terms of the energy reveal the existence of resonant states [41].

The method consists on defining the probability $P(m)$ to find a particle with mass $m$ at the position $z_{0}$ as

$$
P(m)=\frac{\left|\Psi_{m}\left(z_{0}\right)\right|^{2}}{\int_{z_{\min }}^{z_{\max }}\left|\Psi_{m}(z)\right|^{2} d z},
$$

where $z_{\min }$ and $z_{\max }$ stands to domain limits. An extension of this idea was proposed on Ref. [43, where a relative probability is defined as

$$
P(m)=\frac{\int_{z_{a}}^{z_{b}}\left|\Psi_{m}(z)\right|^{2} d z}{\int_{z_{\min }}^{z_{\max }}\left|\Psi_{m}(z)\right|^{2} d z}
$$

evaluated on a narrow range $\left[z_{a}, z_{b}\right]$.

Probability interpretations would be possible for the Sturm-Liouville eigenfunctions by defining the inner product with the weight function included. However, the change of variable $z=z(\rho)$ acts as a domain stretch that improves the treatment for large $c$. By numerical integration, the change of coordinate is plotted in Fig. 9. The analogue quantum potential for the GS model is

$$
U_{G S}(z)=6 z^{-2} .
$$

This potential function does not support bound state and then, there is not any probability to find massive gravitons on the infinitely thin string-like braneworld. Nevertheless, for the string-cigar model, the Schrödinger potential, plotted in Fig. 10, presents an infinite potential well what suggests the possibility of resonant modes. For $c=1.0$ the potential for the string-cigar model behaves as a Coulomb-like potential and, when the parameter $c$ increases, a barrier arises and the potential assumes the usual volcano shape. Massive particles confined on the well may be interpreted as gravitational resonant states (massive quasi-localized gravitons highly coupled to the brane) [10, 39, 40].

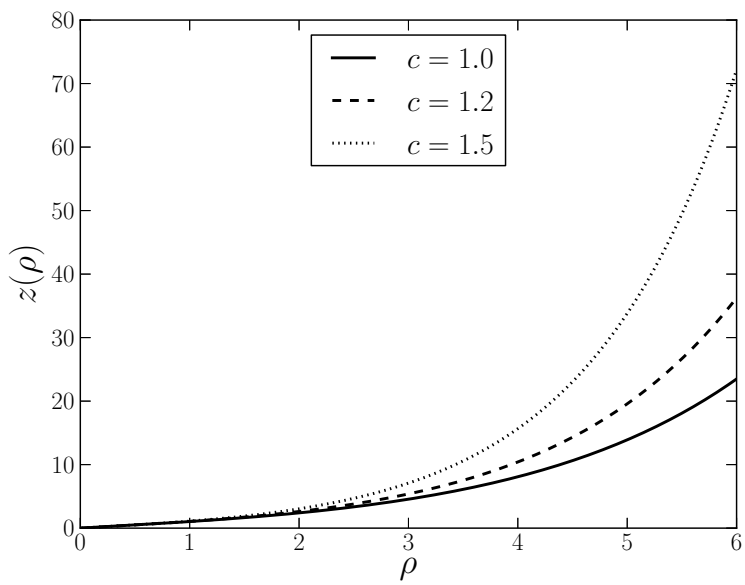

Figure 9: Numerical integral solution of the transformation $z(\rho)$.

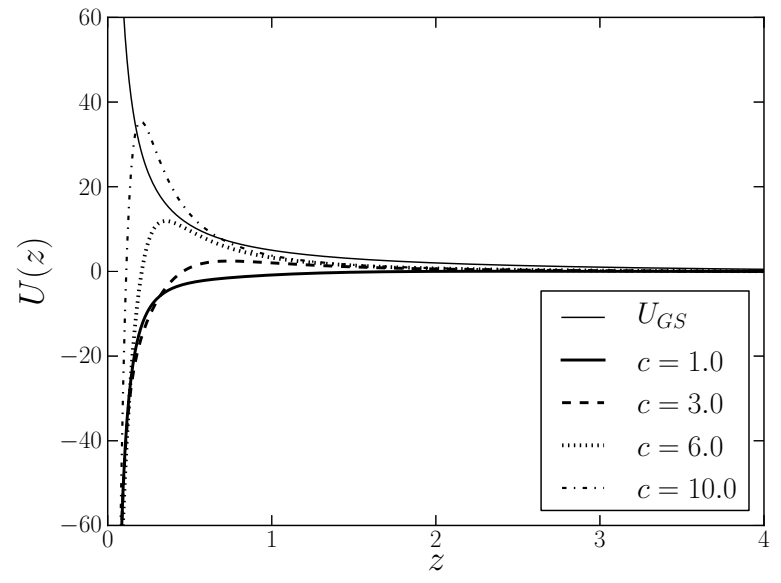

Figure 10: The analogue quantum potential $U(z)$.

We solved the Schrödinger-like equation (27) using the Numerov method [46, 47. The relative probability function (58) is more suitable to detect very narrow resonances if they exist 44. According to the energy-density given in Eq. (50) the brane distribution is $\left[z_{a}, z_{b}\right]=[0.01,0.50]$. The domain is chosen to be $\left[z_{\min }, z_{\max }\right]=[0.01,5.0](10$ times the integration range), for which the plane wave probability would be $P(m)=0.1$ [43]. The position of the resonance peak, where the physical information is stored, does not depend on $z_{\max }$, since it is chosen sufficiently large [44].

The Fig. 11 presents the numerical solution of the relative probability $P(m)$. The first (and largest) peak is revealed for $c=2.9$. The resonant peaks (there is only one for each $c$ ) decrease and become broader with $c$. This means that the resonant massive states lifetime (estimated as $(\Delta m)^{-1}$, where $\Delta m$ is the width at half maximum of the peak [41]) decreases with the cosmological constant.

The Fig. 12 exhibits the solutions of the Schrödinger equation for the masses indicated with the probability peaks. The first solution shows that this particular massive graviton has the highest probability to be found on the brane. 


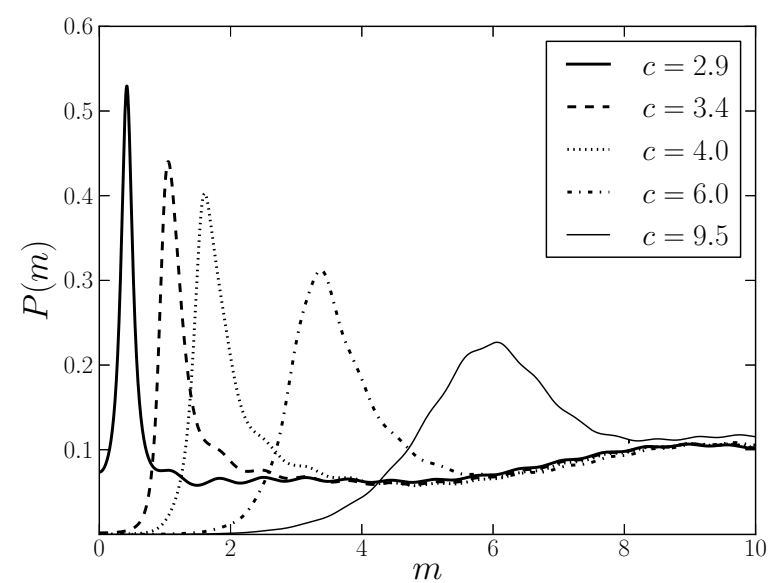

Figure 11: Plot of the relative probability $P(m)$. The peaks reveal the resonant massive states. For $m^{2} \gg U_{\max }$, a plateau is formed at $P=0.1$ corresponding to plane wave regime.

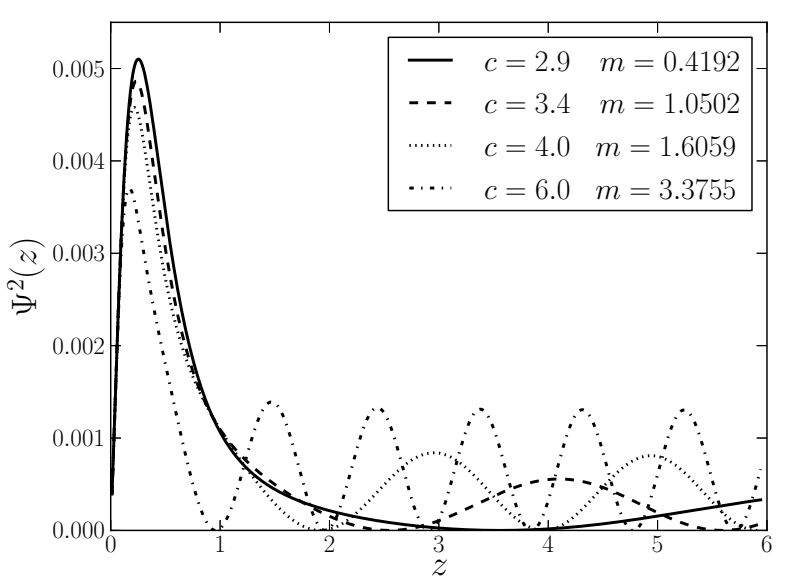

Figure 12: Solutions of the Schrödinger-like equation for the masses corresponding to resonant peaks. The first solution has the highest probability to interact with the brane.

This solution shares a similar behavior to the zero-mode presented in Ref. 27] near the origin, albeit it oscillates asymptotically with large wavelength.

The effects of the brane internal structure on the resonant modes are also shown in Fig. 12. Increasing the value of $c$, which makes the brane thinner (see Fig. 1), enhance the width of the resonance. Therefore, as $\epsilon \rightarrow 0$, the resonant states acquire shorter lifetimes reducing the possibility to massive gravitons result in any physical measurement. This result agrees with the fact that for $c \rightarrow \infty$ the potential barrier tends to the $U_{G S}$, where no massive state is allowed.

\section{CONCLUSIONS AND PERSPECTIVES}

This work displays a study of the gravitational Kaluza-Klein (KK) modes in the Gherghetta-Shaposhnikov (GS) and the string-cigar braneworlds. By the analysis of the energy density, the former can be realised as a thin string-like model whereas the last is regarded as interior and exterior smoothed GS model.

We obtain a new massless mode for the GS model by analysing the radial equation in the Schrödinger approach. It turns out that this massless mode has a bigger amplitude and rate of decay compared with that of the GS model. Further, the transition between the massive and massless modes is not smooth what yields to a mass gap.

We obtained via numerical analysis a complete spectrum of masses and eigenfunctions for both models. Besides the mass gap between the massless mode and the massive modes, both models present a gap separating two regimes. For large values of the discrete index $n$, the spectrum behaves linearly, as predicted by GS model. However, for small $n$, the masses decrease as $1 / m_{n}$. This result can be explained by the feature of the zeros of the Bessel function of the first kind in the two regimes.

Asymptotically, the string-cigar eigenfunctions are similar to GS ones whereas near the brane they behave as the Bessel function of the first kind. It turns out that the amplitude of the modes is bigger in the string-cigar model than in the GS model. Hence, the brane source enhances the modes near the core.

In the GS model, the eigenfunction with a lowest mass resemblances the massless mode with an almost vanishing amplitude, it was referred as transient mode. This transient mode is absent in the string-cigar model what can be regarded as an improvement since the light mode should already have been detected. Then, the resolution of the GS model get rides this transient mode.

The string-cigar model, unlike the GS model, allows the existence of resonant states. Indeed, the conical behavior near the origin yields to a potential well not presented in the GS model. Resonance peaks were found (where the highest one occurs when $c=2.9$ and $m=0.4192$ ) which decrease becoming broader with $c$, meaning that the resonant massive states lifetime decreases with the cosmological constant. The solution for the mass eigenvalue indicated with the highest resonance peak shows that this particular massive graviton has a high probability to be found on the brane.

The results obtained points as perspectives the effects of the gravitational KK modes in a modification of the Newtonian potential. In fact, the higher amplitude of the eigenfunctions at the brane core and the new decreasing 
behavior of the spectrum may lead to interesting modifications in the gravitational potential. Further, Figs. 11 and 12 show that the first resonant peak points out the more expressive massive state to contribute to corrections of the four-dimensional gravity laws.

\section{Acknowledgments}

The authors thank the Fundação Cearense de apoio ao Desenvolvimento Científico e Tecnológico (FUNCAP), the Coordenação de Aperfeiçoamento de Pessoal de Nível Superior (CAPES), the Conselho Nacional de Desenvolvimento Científico e Tecnológico (CNPq) and the Instituto Federal do Ceará (IFCE) for financial support.

[1] L. Randall and R. Sundrum, Phys. Rev. Lett. 83, 3370 (1999).

[2] L. Randall and R. Sundrum, Phys. Rev. Lett. 83, 4690 (1999).

[3] N. Arkani-Hamed, S. Dimopoulos and G. R. Dvali, Phys. Lett. B 429, 263 (1998).

[4] Th. Kaluza, Sitzungsber. Preuss. Akad. Wiss. Berlin, K1 966 (1921).

[5] O. Klein, Z. Phys. 37 (1926) 895; Arkiv. Mat. Astron. Fys. B 34A (1946).

[6] K. Akama, Lect. Notes Phys. 176, 267 (1982).

[7] V. A. Rubakov and M. E. Shaposhnikov, Phys. Lett. B 125, 136 (1983).

[8] V. A. Rubakov and M. E. Shaposhnikov, Phys. Lett. B 125, 139 (1983).

[9] M. Visser, Phys. Lett. B 159, 22 (1985).

[10] Martin Gremm, Phys. Lett. B 478, 434-438 (2000).

[11] W. Israel, Phys. Rev. D 15, 935 (1977); A. Vilenkin, Phys. Rev. Lett. 46, 171169 (1981); J. Gott III, Astrophys. J., 288 422 (1985); W. A. Hiscock, Phys. Rev. D 31, 3288 (1985); R. Geroch and J. Traschen, Phys. Rev. D 36 (1987).

[12] I. Navarro and J. Santiago, JHEP 0502, 007 (2005); I. Navarro, JCAP 0309, 004 (2003); S. Kanno and J. Soda, JCAP 0407, 002 (2004); G. Kofinas, Class. Quant. Grav. 22, L47 (2005); G. Kofinas, Phys. Lett. B 633, 141 (2006).

[13] R. Gregory, Phys. Rev. Lett. 59, 740 (1987); R. Gregory, Phys. Lett. B 215, 663 (1988); A. G. Cohen and D. B. Kaplan, Phys. Lett. B 215, 67 (1988); G. Gibbons, M. Ortiz and F. Ruiz, Phys. Rev. D 39, 61546 (1989); V. P. Frolov, W. Israel and W. G. Unruh, Phys. Rev. D 39, 1084 (1989); R. Gregory, Phys. Rev. D 54, 4955 (1996); M. Christensen, A. L. Larsen and Y. Verbin, Phys. Rev. D 60, 125012 (1999).

[14] A. G. Cohen and D. B. Kaplan, Phys. Lett. B 470, 52 (1999).

[15] R. Gregory, Phys. Rev. Lett. 84, 2564 (2000).

[16] I. Olasagasti and A. Vilenkin, Phys. Rev. D 62, 044014 (2000).

[17] M. Giovannini, H. Meyer and M. E. Shaposhnikov, Nucl. Phys. B 619, 615 (2001).

[18] E. Ponton and E. Poppitz, JHEP 0102, 042 (2001).

[19] T. Gherghetta and M. E. Shaposhnikov, Phys. Rev. Lett. 85, 240 (2000).

[20] I. Oda, Phys. Lett. B 496, 113 (2000).

[21] I. Oda, Phys. Rev. D 62, 126009 (2000).

[22] Y. X. Liu, L. Zhao and Y. S. L. Duan, JHEP 04, 097 (2007).

[23] A. Kehagias, Phys. Lett. B 600, 133 (2004).

[24] J. Garriga and M. Porrati, JHEP 0408, 028 (2004).

[25] M. Gogberashvili, P. Midodashvili and D. Singleton, JHEP 0708, 033 (2007).

[26] B. de Carlos and J. M. Moreno, JHEP 0311, 040 (2003).

[27] J. E. G. Silva, V. Santos and C. A. S. Almeida, Class. Quant. Grav. 30, 025005 (2013).

[28] B. Chow, P. Lu and L. Ni, Hamilton's Ricci flow, Science Press, 2006.

[29] R. S. Hamilton, The Ricci flow on surfaces, Contemporary Mathematics 71 (1988), 237-261.

[30] D. Friedan, Phys. Rev. Lett. 45, 1057 (1980); T. Oliynyk, V. Suneeta and E. Woolgar, Nucl. Phys. B 739, 441 (2006); A. A. Tseytlin, Phys. Rev. D 75, 064024 (2007); T. Oliynyk, V. Suneeta and E. Woolgar, Phys. Rev. D 76, 045001 (2007).

[31] M. Headrick and T. Wiseman, Class. Quant. Grav. 23, 6683 (2006).

[32] D. Xianzhe and L. Ma, Comm. Math. Phys. 274, Number 1, 65-80 (2007).

[33] N. Lashkari and A. Maloney, Class. Quant. Grav. 28, 105007 (2011).

[34] P. P. Orth, P. Chandra, P. Coleman and J. Schmalian, Phys. Rev. Lett. 109, 237205 (2012).

[35] S. Randjbar-Daemi and M. E. Shaposhnikov, Phys. Lett. B 491, 329 (2000).

[36] J. E. G. Silva and C. A. S. Almeida, Phys. Rev. D 84, 085027 (2011).

[37] P. Tinyakov and K. Zuleta, Phys. Rev. D 64, 025022 (2001).

[38] G. Watson, A treatise on the theory of the Bessel functions, Cambridge University Press, 1996.

[39] C. Csaki, J. Erlich, T. J. Hollowood and Y. Shirman, Nucl. Phys. B 581, 309 (2000).

[40] C. Csaki, J. Erlich, and T. J. Hollowood, Phys. Rev. Lett. 84, 5932 (2000).

[41] C. A. S. Almeida, M. M. Ferreira, Jr., A. R. Gomes and R. Casana, Phys. Rev. D 79, 125022 (2009).

[42] A. R. Gomes, "Gravity on the Bloch Brane", arXiv:hep-th/0611291 (2006). 
[43] Yu-Xiao Liu, Jie Yang, Zhen-Hua Zhao, Chun-E Fu, and Yi-Shi Duan, Phys. Rev. D 80, 065019 (2009).

[44] W. T. Cruz, L. S. J. Sousa, R. V. Maluf, C. A. S. Almeida, Phys. Lett. B 730, 314-319 (2014).

[45] Pierluigi Amodio and Giuseppina Settanni, J. Numer. Anal. Indust. Appl. Math vol.6 no. 1-2, 2011, pp.1-13.

[46] B.V. Numerov, Monthly Notices of the Royal Astronomical Society, Vol. 84, p.592-592 (1924).

[47] B.V. Numerov, Astronomische Nachrichten, Vol. 230, p.359 (1927). 